\title{
Hedgehog signaling can enhance glycolytic ATP production in the
}

\section{Drosophila wing disc}

Ioannis Nellas ${ }^{1}$, K. Venkatesan Iyer ${ }^{1,2}$, Juan M. Iglesias-Artola ${ }^{1}$, André Nadler ${ }^{1}$, Natalie A. Dye $^{1,3,4 *}$ and Suzanne Eaton ${ }^{1,4, \dagger}$

*Corresponding author: natalie_anne.dye@tu-dresden.de

${ }^{1}$ Max Planck Institute for Molecular Cell Biology and Genetics, Dresden, Germany

${ }^{2}$ Current address: Department of Mechanical Engineering, Indian Institute of Science, Bangalore, 560012, India

${ }^{3}$ Mildred Scheel Nachwuchszentrum (MSNZ) P2, Medical Faculty, Technische Universität Dresden, Germany

${ }^{4}$ Excellence Cluster, Physics of Life, Technische Universität Dresden, Germany

${ }^{\dagger}$ Deceased, July 2019

Running Title (40 characters): Hedgehog can enhance ATP production

\section{ABSTRACT:}

Energy production and utilization is critically important for animal development and growth. How it is regulated in space and time during tissue growth remains largely unclear. Toward this end, we used a FRET-based adenosine triphosphate (ATP) sensor to dynamically monitor ATP levels across a growing tissue, using the Drosophila wing disc. We discovered that steady-state levels of ATP are spatially uniform across the wing pouch. Pharmacologically inhibiting oxidative phosphorylation, however, reveals spatial heterogeneities in metabolic behavior, whereby signaling centers at compartment boundaries produce more ATP from glycolysis than the rest of the tissue. Genetic perturbations indicate that the conserved Hedgehog (Hh) signaling pathway can enhance ATP production by glycolysis. Collectively, our work reveals a positive feedback loop between Hh signaling and energy metabolism, advancing our understanding of the connection between conserved developmental patterning genes and energy production during animal tissue development.

\section{KEYWORDS:}

Hedgehog, glycolysis, ATP, Drosophila, metabolism 


\section{INTRODUCTION:}

The regulation of energy production and utilization is critically important for the growth of living organisms. The energetic currency ATP is produced by the breakdown of glucose to pyruvate during glycolysis (yielding 2 ATPs), followed by the oxidation of pyruvate through the TCA cycle and oxidative phosphorylation (OxPhos) under normoxic conditions $(\sim 36$ ATPs). In the absence of sufficient oxygen, pyruvate can be converted to lactate and secreted. Even under normoxic conditions, increased glucose uptake can result in the secretion of excess pyruvate as lactate, a phenomenon called "aerobic glycolysis". This behavior is thought to allow intermediate metabolites from the glycolytic pathway and the TCA cycle to be channeled to other pathways to rapidly produce macromolecular precursors (nucleotides, amino acids, and lipids), which are also required for growth (Lunt and Vander Heiden, 2011).

Tissue growth during animal development also involves morphogens - secreted signals that pattern gene expression across a tissue in a concentration-dependent manner (Rogers and Schier, 2011). Hedgehog is one such morphogen that promotes the growth and patterning of many different tissues during development and also regulates many processes during adult homeostasis (Ingham and McMahon, 2001; Petrova and Joyner, 2014). One of the vertebrate Hh homologs, Sonic Hedgehog (SHH), has been shown to promote aerobic glycolysis in fat cells and in cerebellar granule neuron precursors (Teperino et al., 2012; Gershon et al., 2013; Di Magno et al., 2014). Thus, it is interesting to investigate how spatial gradients of Hh that form during tissue development may affect metabolism and energy production. Recently developed live, fluorescent biosensors have made it possible to address this question by enabling the monitoring of metabolites at high spatial-temporal resolution in living cells and tissues (Tsuyama et al., 2013; Bulusu et al., 2017; Greenwald, Mehta and Zhang, 2018; Volkenhoff et al., 2018).

Here, we study the spatial dynamics of ATP levels in the Drosophila wing disc, a growing tissue that has been a powerful model system for studying principles of morphogen signaling and developmental tissue growth (Hariharan, 2015; Beira and Paro, 2016). The patterns of morphogen signaling are well characterized, easy to visualize in the flat "pouch" region, and able to be genetically perturbed with spatial and temporal control. We exploit these properties to study how Hh may influence energy production. Previous work indicates that OxPhos is a major provider of ATP in the wing disc (Spannl et al., 2020) and that little aerobic glycolysis is normally observed in the tissue (De La Cova et al., 2014; Wang et al., 2016; Bawa et al., 2020). Nonetheless, glycolytic genes are expressed during normal wing disc development (Dye et al., 2017; Spannl et al., 2020) and their genetic depletion results in a mild but consistent reduction of steady state ATP levels and undergrowth of the tissue (Spannl et al., 2020). Furthermore, loss of glycolytic gene expression affects the plasma membrane potential, causing reduced uptake of Hh-inhibitory lipids and thereby an upregulation of Hh signaling. Thus, glycolysis has a function in maintaining Hh signaling and tissue growth in the wing disc. Whether Hh in turn affects energy production in this tissue is not known.

Using a recently developed FRET-based ATP sensor (Tsuyama et al., 2013), we dynamically monitored ATP levels across the wing disc tissue. We find that OxPhos inhibition reveals higher glycolytic ATP production in the Hh signaling domain and that genetic modification of the pathway can influence ATP production from glycolysis. Altogether, our 
bioRxiv preprint doi: https://doi.org/10.1101/2021.09.11.459911; this version posted September 12, 2021. The copyright holder for this preprint (which was not certified by peer review) is the author/funder, who has granted bioRxiv a license to display the preprint in perpetuity. It is made available under aCC-BY 4.0 International license.

work establishes positive feedback between morphogen signaling and metabolic activity during developmental tissue growth.

\section{RESULTS and DISCUSSION:}

\section{OxPhos inhibition reveals spatially heterogeneous metabolism in the wing pouch}

We monitored ATP levels across the wing pouch in live explants using a ubiquitously expressed FRET-based ATP sensor, ubi-AT1.03NL (Fig 1A) (Tsuyama et al., 2013). Steady state levels of ATP are similar throughout the wing pouch and decline considerably upon OxPhos inhibition with antimycin A (Fig 1C, (Spannl et al., 2020)). After $2 \mathrm{hr}$ of treatment with antimycin A, ATP levels fall close to the lower detection limit of the sensor (as determined by the use of the ATP-insensitive sensor ubi-AT1.03RK, Fig S1B \& E-F), whereas ATP levels in control wing discs remain stable over this time period (Fig S1 C-D). Interestingly, a transient pattern emerges in the wing pouch during OxPhos inhibition (Fig 1C). ATP levels drop slightly slower in two perpendicular stripe regions, corresponding to the Dorsal-Ventral (DV) boundary and an area just anterior to the Anterior-Posterior (AP) boundary (Fig S1I-J). These areas are known growth "organizer" regions, with high signaling activity in the Wingless/Notch and Hedgehog pathways, respectively (Fig 1B).

To quantitatively compare the kinetics of ATP depletion in different regions, we locally measured FRET efficiency over time during OxPhos inhibition (Fig 1D, G, Fig S1G-J). To describe the kinetic data, we used a four-parametric logistic curve that fits the data well. This fitting allows us to estimate the initial and final state of the FRET efficiency, the time needed to reach half of the FRET efficiency (half-life, units of time) and the slope of the curve at the half-life (Hill coefficient, unitless). We use the half-life and Hill coefficient to compare the kinetics of ATP depletion in different tissue regions (see Fig S1H and methods). Since the FRET efficiency decreases from a high state to a lower state, the Hill coefficient is negative. Consequently, a lower Hill coefficient corresponds to a steeper transition from the initial to the final state. In addition, lower values of half-life correspond to faster kinetics of ATP depletion. We compare relative changes in these kinetic fit parameters across different regions of the tissue. We find larger Hill coefficient and half-life values for the AP and DV boundary regions than for the rest of the pouch (Fig 1E-F, H-I). Thus, the AP and DV organizer regions have significantly slower kinetics of ATP depletion upon OxPhos inhibition than the rest of the wing pouch. 
A

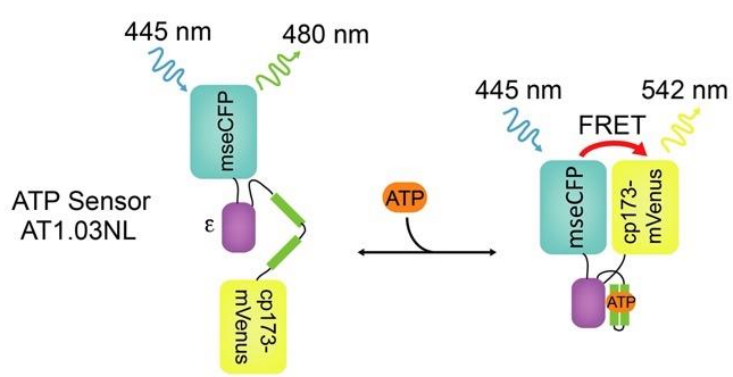

C

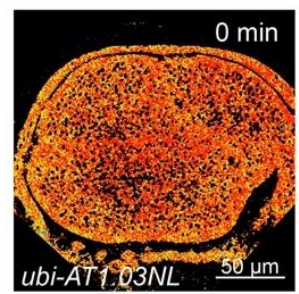

D

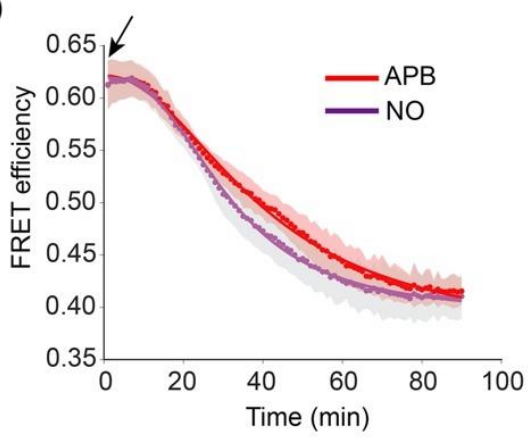

G

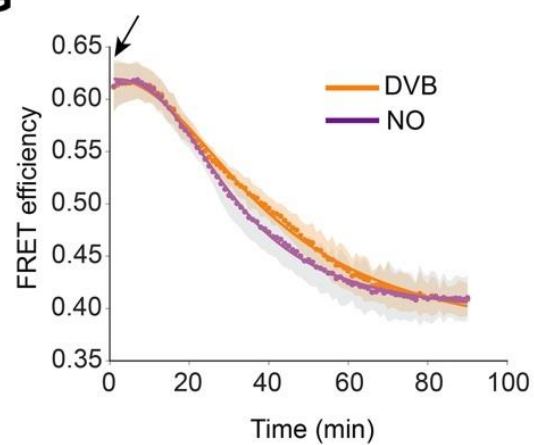

E

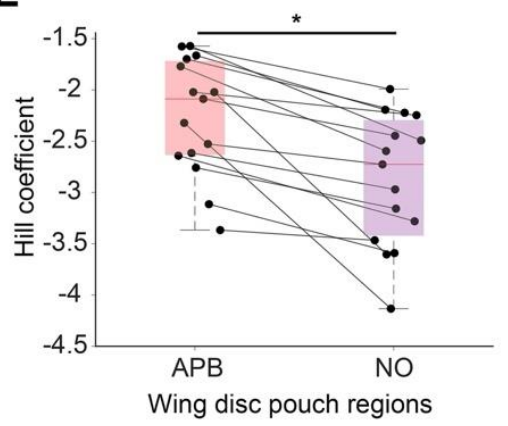

H

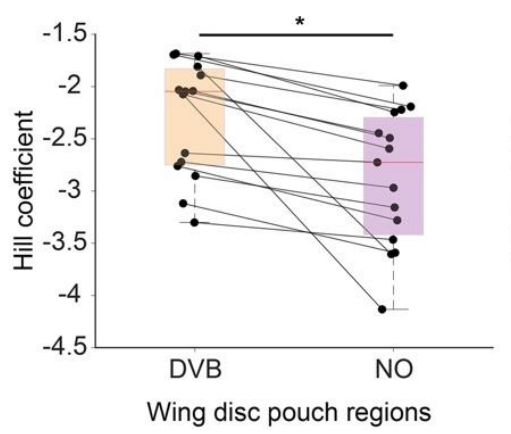

B Wing disc organizer regions
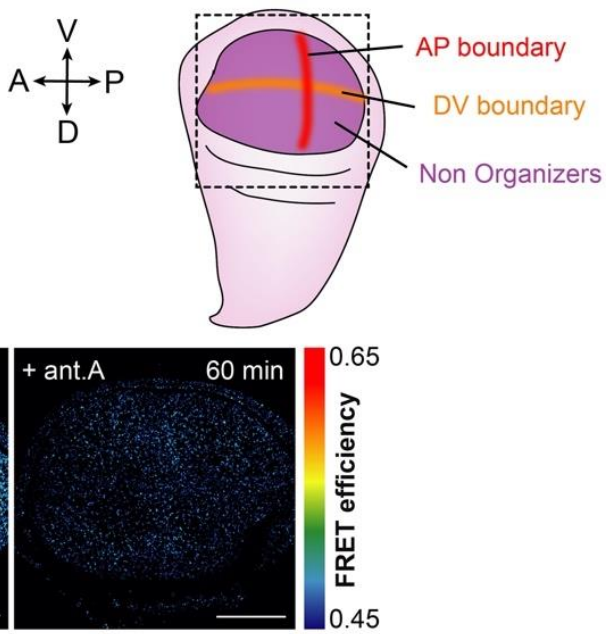

$\mathbf{F}$

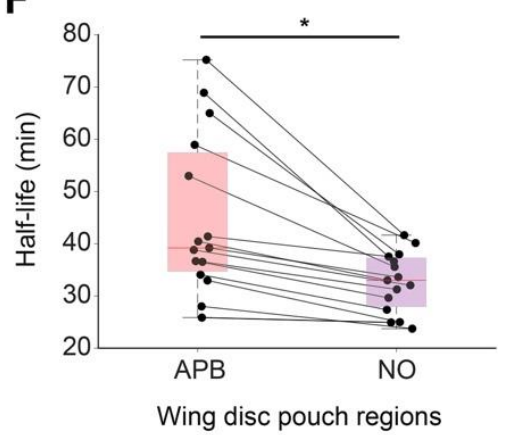

I

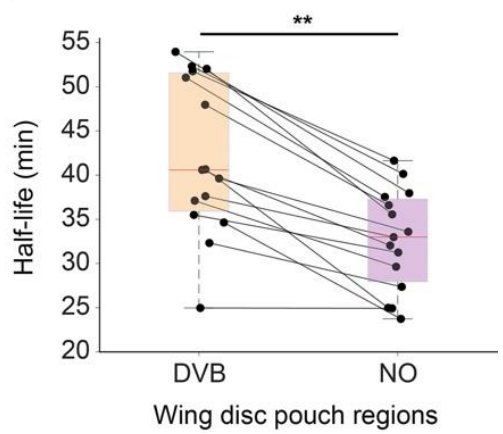

Figure 1: OxPhos inhibition depletes ATP levels slower at the organizers than elsewhere in the 2013). Binding of ATP leads to a conformational change that brings the two fluorophores (donor and acceptor) in close proximity, resulting in FRET. (B) Schematic representation of the wing disc highlighting organizer and non-organizer regions. (C) Timelapse montage of ATP sensor FRET efficiency across the wing pouch after $10 \mu \mathrm{M}$ antimycin A (ant.A) addition. (D, G) Mean FRET efficiency measured over time in the (D) AP boundary (APB) or (G) DV boundary (DVB) and nonorganizer regions (NO). Shaded regions indicate standard deviation (SD); solid lines illustrate a fit to the mean data. (E-F, H-I) Fit parameters of individual time traces for AP boundary and non-organizer regions (E-F) or DV boundary and NO regions (H-I). Black lines connect the corresponding regions of the same disc. $*=$-value $<0.05, * *=p$-value $<0.01$ using a Kruskal-Wallis test $(\mathrm{n}=15)$. Black arrows indicate the addition of the drug, and small colored dots represent the averages at each timepoint. 
bioRxiv preprint doi: https://doi.org/10.1101/2021.09.11.459911; this version posted September 12, 2021. The copyright holder for this preprint (which was not certified by peer review) is the author/funder, who has granted bioRxiv a license to display the preprint in perpetuity. It is made available under aCC-BY 4.0 International license.

\section{Organizer regions can enhance glycolytic ATP production}

ATP levels in organizer regions may decline slower upon OxPhos inhibition either because these regions consume ATP slower or produce more ATP from glycolysis (which is unaffected by antimycin A). To distinguish between these possibilities, we looked at the effect of simultaneously inhibiting glycolysis and OxPhos. We found that combining the OxPhos inhibitor antimycin A with the glycolytic inhibitors 3-bromo-pyruvate (3BP) and 2-deoxy-Dglucose (2DG) significantly alters the pattern observed with antimycin A alone (Fig 2A): ATP levels decay in organizer and non-organizer regions with similar kinetics (Fig 2B-D). Half-life values are indistinguishable (Fig 2D), and the Hill coefficient is slightly lower in the organizer regions than elsewhere in the tissue (Fig 2C). The latter indicates a slightly faster depletion of ATP in organizers, suggesting a higher ATP consumption rate. Thus, we conclude that organizer regions obtain more ATP from glycolysis during OxPhos inhibition than the rest of the wing pouch.

Note that the kinetics of ATP depletion throughout the wing pouch are faster upon inhibition of both OxPhos and glycolysis than upon inhibition of OxPhos alone, as indicated by a comparison of the Hill coefficients in Figs 1 and 2: -5.5 in non-organizers and -5.9 in organizers in all drugs (Fig 2C), compared to -2.7 in non-organizers and around -2.1 in both AP and DV boundaries in antimycin alone (Fig 1E, H). This finding indicates that upon OxPhos inhibition, glycolysis still generates ATP in the entire wing pouch but more so in the organizer regions. We were unable, however, to detect any difference in ATP levels with the FRET reporter after $2 \mathrm{hr}$ of exposure to glycolytic inhibitors 3BP and 2DG alone or in combination, even when used at very high concentrations (Fig 2E-H, Fig S2D-I). Similarly, using a luciferase-based assay to measure ATP levels in whole wing discs, we did not find any significant effects of glycolytic inhibition (Fig S2A-B), even as OxPhos inhibition induced a rapid depletion of ATP (Fig S2C). We interpret these results to mean that most of the ATP in the wing disc is generated by OxPhos and that OxPhos can compensate for the $2 \mathrm{hr}$ pharmacological inhibition of glycolysis. Nonetheless, the depletion of glycolytic enzymes by RNAi over several days results in a reduction of steady state ATP levels (Spannl et al., 2020). It remains possible that the FRET-ATP sensor is saturated and therefore cannot detect small drops in ATP upon glycolytic inhibition alone. We do not think this a likely scenario, however, given that most of our experiments start with an ATP FRET efficiency of only $\sim 0.6$, but values of up to 0.7 can be observed in the same tissue under similar conditions, suggesting that the sensor can read higher concentrations of ATP. 


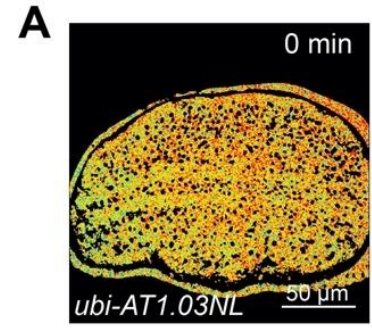

B

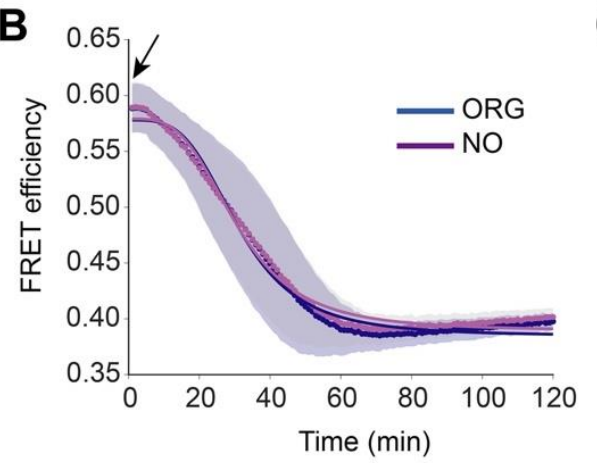

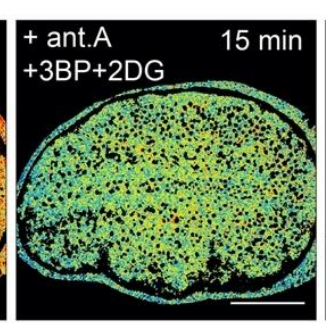

C

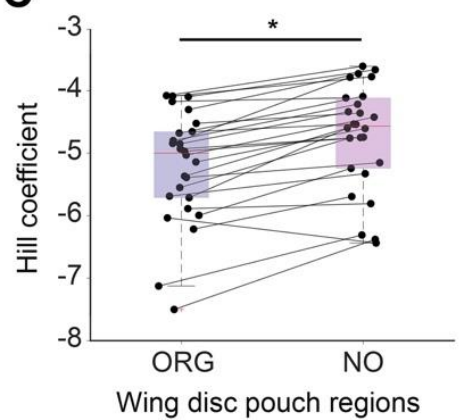

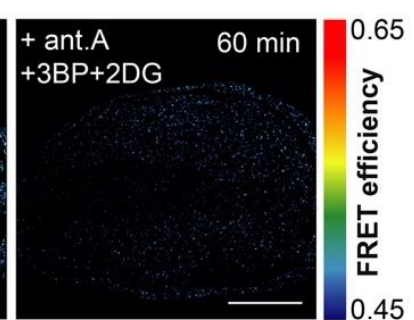

D

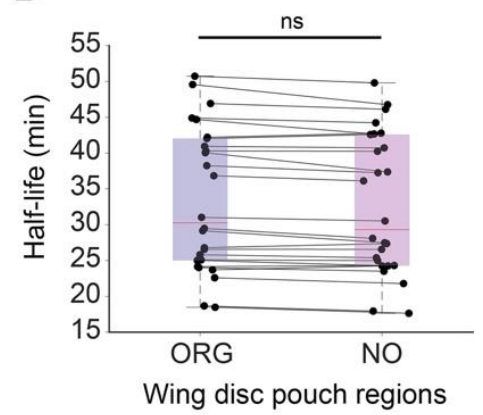

$\mathbf{E}$

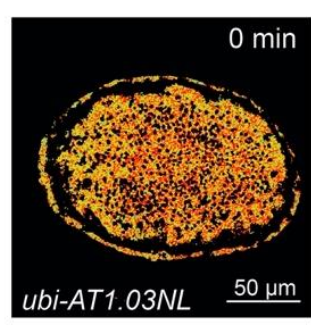

$\mathbf{F}$

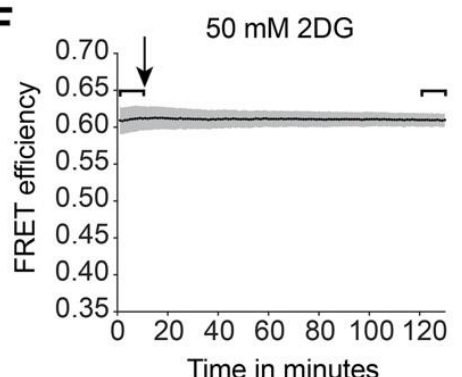

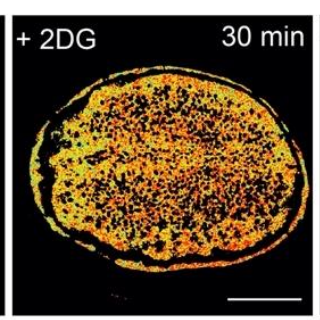

G
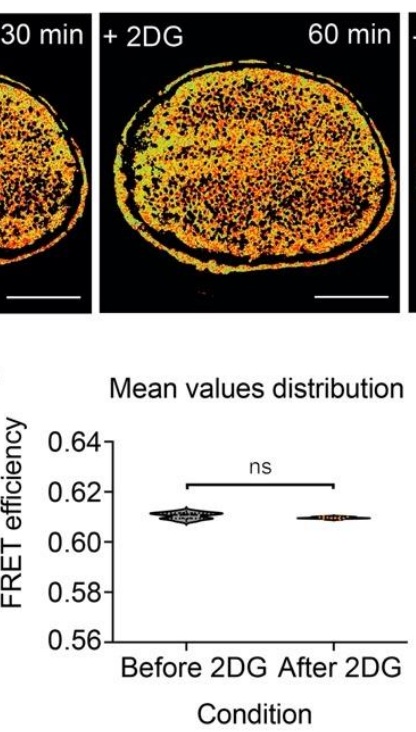
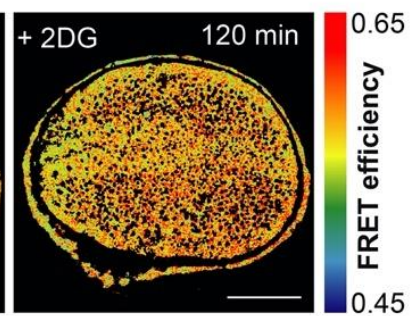

H

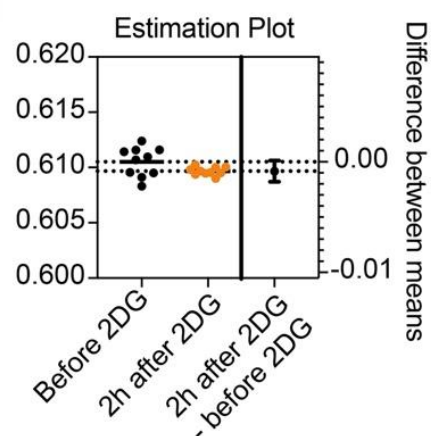

Figure 2: Glycolysis is required for spatial heterogeneity in ATP levels upon OxPhos inhibition. (A) Timelapse montage of ATP sensor FRET efficiency distribution after combined addition of $10 \mu \mathrm{M}$ antimycin A (ant.A), $100 \mu \mathrm{M}$ 3-Bromopyruvate (3BP), and $50 \mathrm{mM}$ 2-deoxy-D-glucose (2DG). (B) Mean FRET efficiency of organizer (ORG) regions and non-organizer (NO) regions analyzed over time. Shaded regions indicate SD; blue and violet lines illustrate a fit of the mean data. (C, D) Fit parameters of individual time traces for organizer and non-organizer regions. Black lines connect the corresponding regions of the same disc. $*=$ p-value $<0.05$, ns $=$ not significant $\mathrm{p}$-value using a Kruskal-Wallis test $(n=26)$. (E) Time lapse montage of ATP sensor FRET efficiency in the wing pouch after 2DG addition. (F) Mean FRET efficiency time trace of the entire wing pouch; gray shade indicates SD. Brackets include the mean FRET values before and 2h after 2DG addition (1-10 min and 121-130 min respectively) whose distribution was compared using $(\mathrm{G})$ an unpaired t-test (ns = not significant $\mathrm{p}$-value, $\mathrm{n}=17$ ) including $(\mathrm{H})$ Welch's correction and estimation plot ( $\mathrm{n}=17$ for each group). Black arrows indicate the addition of the drugs, and small colored dots represent the averages at each timepoint. 
183

184

185

186

187

188

189

190

191

192

193

194

195

196

197

198

199

200

201

202

203

204

205

206

207

208

209

210

211

212

213

214

215

216

217

218

219

220

221

222

223

224

225

226

\section{Hedgehog signaling enhances ATP production by glycolysis upon OxPhos inhibition}

Our results suggest that the morphogen signaling that defines the organizer regions can influence energy production in the wing disc. It has been previously shown that Notch signaling, which is normally localized on either side of the DV boundary, can upregulate glycolysis (Slaninova et al., 2016), which is consistent with our data. The Hh pathway organizes growth and patterning along the AP axis, but its contribution to energy production has not been explored.

In the wing disc, Hh is expressed in the posterior compartment and travels to the anterior compartment, where it is received by the membrane protein, Patched (Ptc) (Basler and Struhl, 1994; Tabata and Kornberg, 1994). In the absence of Hh, Ptc represses the 7-pass signal transducer Smoothened (Denef et al., 2000). Hh binding to Ptc relieves this repression, allowing the Gli-family transcription factor Cubitus interruptus (Ci) to activate target gene expression.

To test whether Hh could affect energy production in the wing disc, we genetically activated and repressed the pathway in a temporally and spatially controlled manner using the Gal4/UAS system (Brand and Perrimon, 1993; Del Valle Rodríguez, Didiano and Desplan, 2012) and examined the effect on the kinetics of ATP depletion during pharmacological inhibition of glycolysis and OxPhos. We used apterous-Gal4 combined with tub-Gal80 $0^{\text {ts }}$ $\left(a p G a l^{t s}\right)$ to temporally perturb Hh signaling only in the dorsal compartment of the wing disc, leaving the ventral compartment as an internal control (Fig 3A). In the mock-treated apGal ${ }^{\text {ts }}$ genetic background alone (without a UAS construct), there is no difference in the kinetics of ATP depletion upon OxPhos inhibition between dorsal and ventral compartments (Fig S3).

We used ap Galts to overactivate the Hh pathway dorsally by inducing RNAi against Ptc, the Hh receptor that represses the pathway in the absence of ligand. Ptc is normally expressed in the anterior compartment, with a peak near the AP boundary (Fig 3B, S1J, S5A). Dorsal downregulation of Ptc does not affect the spatial pattern of steady state ATP levels in the wing disc but strikingly alters the pattern of metabolic activity in response to antimycin A (Fig 3C-I, Fig S4A-F). We observe a significantly longer half-life value for ATP depletion in the anterior region of the dorsal compartment (DA in Fig 3F, half-life of $96.7 \mathrm{~min}$ ) compared to the anterior region of the ventral compartment (VA in Fig 3F, half-life of $33 \mathrm{~min}$ ), which is not affected by apGal ${ }^{t s}$. The half-life for the DA region is also significantly longer than that of the posterior region of the dorsal compartment (DP, half-life of $33 \mathrm{~min}$, Fig 3I). This difference can be explained by the fact that Ptc is normally only expressed in the anterior compartment. To confirm that this effect is cause by more ATP being produced by glycolysis, we again used the combination of OxPhos and glycolytic inhibitors (antimycin A, 3BP, and 2DG). Inhibiting both metabolic pathways eliminated the spatial differences in kinetics of ATP decline (Fig S4G$\mathrm{K})$. Thus, OxPhos inhibition reveals that overactivation of Hh signaling can enhance glycolytic ATP production.

Loss of Patched will increase the expression of another important growth regulator and Hh target gene, Decapentaplegic (Dpp). Dpp is produced in the anterior compartment in response to $\mathrm{Hh}$ signaling, but it is secreted and migrates bidirectionally through the tissue to promote growth and proliferation on both the anterior and posterior sides (Affolter and Basler, 2007; Restrepo, Zartman and Basler, 2014). Interestingly, in apGal ${ }^{t s}>P t c^{R N A i}$ discs, we found increased proliferation throughout the dorsal compartment, with no statistical difference 
between the anterior and posterior sides (Fig S5), consistent with an upregulation of Dpp. It is unlikely, however, that Dpp mediates the effect of Hh overactivation on glycolytic ATP production, as we only see an effect of $P t c^{R N A i}$ in the Hh-receiving anterior compartment, where

230 Ptc is normally expressed (Fig 3B-I, Fig S4D-F).

231

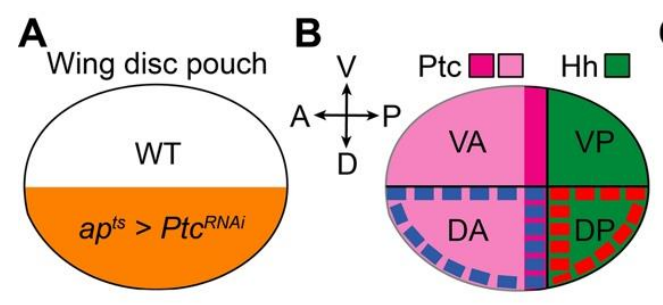

D

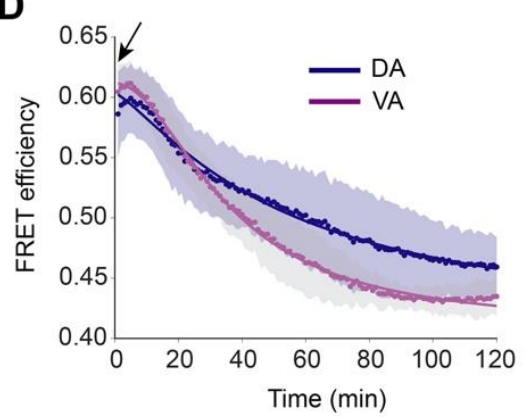

G

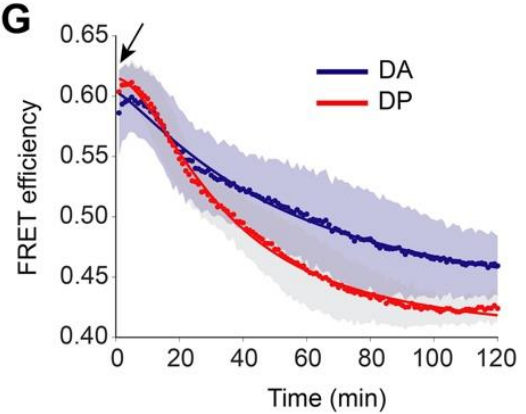

E

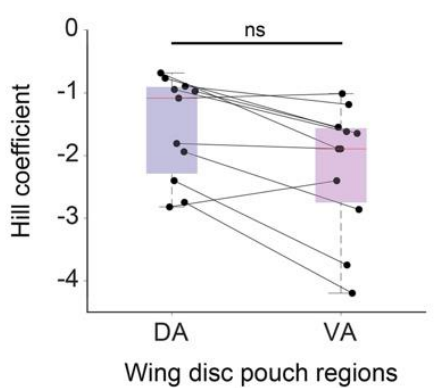

H

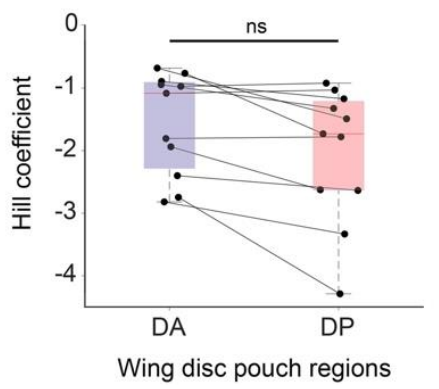

$\mathbf{F}$

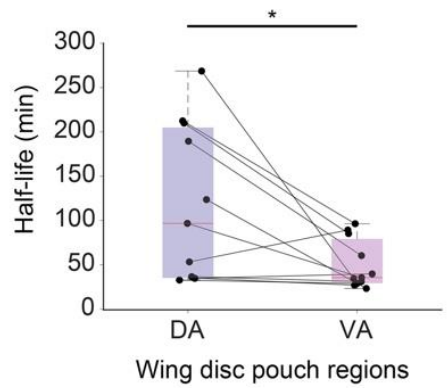

|

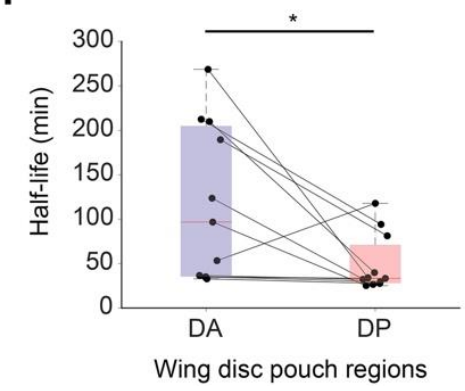

Figure 3. Upregulation of Hh activity enhances glycolytic ATP production upon OxPhos inhibition. (A) Schematic representation of Ptc knockdown in the dorsal compartment. (B) Schematic showing the expression pattern of Ptc and $\mathrm{Hh}$ in the wing disc. Ptc is expressed in the anterior compartment (light magenta) and its highest expression is at a narrow stripe right next to the AP boundary (dark magenta). (C) Timelapse montage of ATP sensor FRET efficiency in the wing pouch after $10 \mu \mathrm{M}$ antimycin A (ant.A) addition in $a p G a l^{t s}>P t c^{R N A i}$ wing discs. (D, G) Mean FRET efficiency analyzed over time in the (D) dorsal anterior (DA) and ventral anterior (VA) sub-compartments or the (G) dorsal anterior (DA) and dorsal posterior (DP) sub-compartments of $a p G a l^{t s}>P t c^{R N A i}$ wing discs. Shaded regions indicate SD; solid lines illustrate a fit of the mean data. (E-F, H-I) Fit parameters of individual time traces for DA and VA compartments (E-F) or DA and DP compartments (H-I). Black lines connect the corresponding regions of the same disc. $*=$ p-value $<0.05$, ns $=$ not significant $\mathrm{p}-$ value using a Kruskal-Wallis test $(n=11)$. Black arrows indicate the addition of the drug and small colored dots represent the average values in each time point.

To further test whether Hh pathway activity can regulate glycolysis in wing discs, we reduced Hh pathway activity by overexpressing a dominant negative form of the downstream Hh-responsive transcription factor, Cubitus interruptus $\left(\mathrm{Ci}^{\mathrm{DN}}\right.$, also named $\mathrm{Ci}^{\text {Cell }}$ in (Méthot and 
Basler, 1999)) (Fig 4). We used ap Gal ${ }^{\text {ts }}$ to induce $\mathrm{Ci}^{\mathrm{DN}}$ in the dorsal compartment of the wing disc. This perturbation has the opposite effect of Ptc ${ }^{R N A i}$ : upon loss of Hh activity, ATP levels decline faster during OxPhos inhibition (Fig 4C-E). Both the Hill coefficient and half-life values are significantly lower in the dorsal compartment than in the ventral (Hill coefficient $=$ -4.5 for dorsal vs -1.8 for ventral; half-life $=32 \mathrm{~min}$ for dorsal, $42 \mathrm{~min}$ for ventral). This result suggests that down-regulation of Hh pathway activity using a dominant negative version of its downstream transcription factor reduces ATP production from glycolysis. Note that this perturbation, unlike $P t c^{R N A i}$, affects both the anterior and posterior sides of the dorsal compartment (Fig 4B). Although Ci is only expressed in the anterior compartment in wild type discs, here we are forcing the expression of a dominant negative construct in the entire dorsal domain, and therefore it is not unexpected to see an effect on both sides of the AP boundary.

Taken together, our results from reciprocal genetic perturbations strongly suggest that Hh can positively affect glycolytic ATP production, at least upon inhibition of OxPhos. Given that glycolysis is required for normal levels of Hh signaling (Spannl et al., 2020), these new data indicate that there exists a positive feedback loop between $\mathrm{Hh}$ and glycolysis that could be part of a homeostatic mechanism coupling energy with developmental patterning and growth. Deciphering the underlying mechanism by which Hh promotes glycolysis in this model tissue is an important open question.

In summary, we show here that OxPhos inhibition reveals spatial heterogeneity in energy production in the wing pouch and that genetic perturbation experiments support a role for Hh in promoting ATP production by glycolysis. This work provides a foundation for new studies exploring how morphogen signaling and energy production interact to promote growth during tissue development.

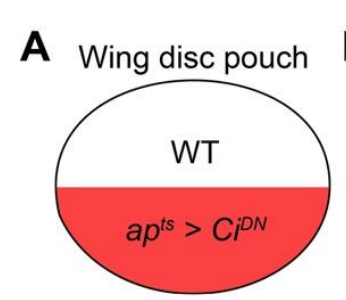

C
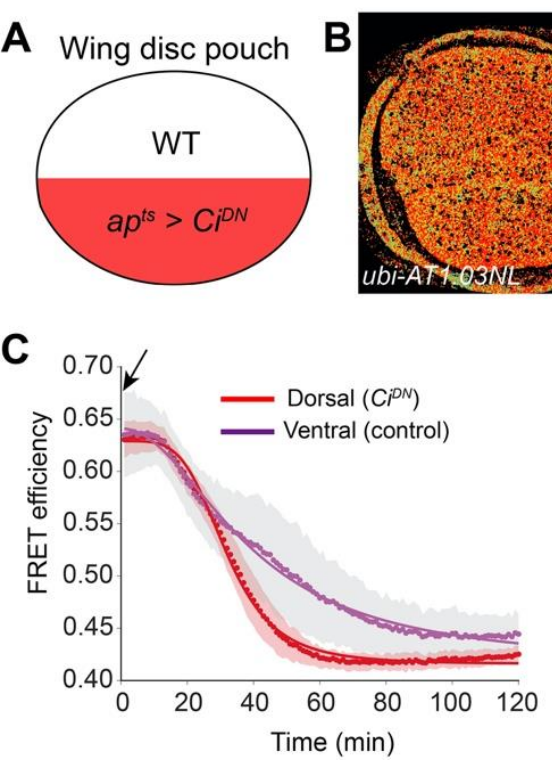

D

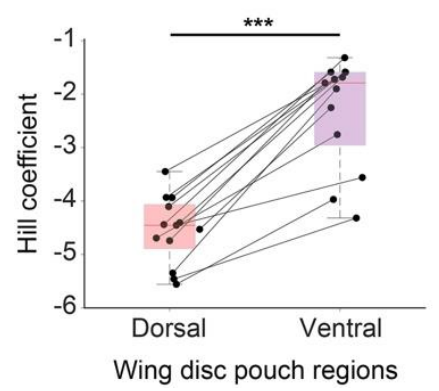

E

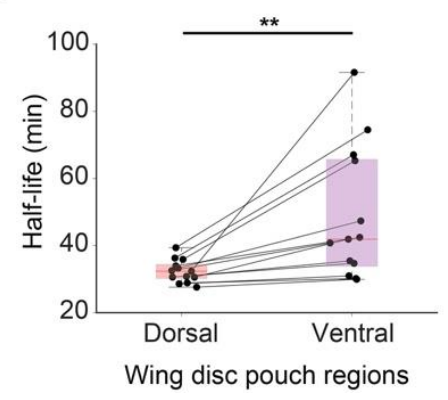

Figure 4. Downregulation of Hedgehog activity reduces glycolytic ATP production upon OxPhos

inhibition. (A) Schematic showing the dorsal expression of the dominant negative $\mathrm{Ci}$ allele $\left(\mathrm{Ci}^{\mathrm{DN}}\right)$. (B) Timelapse montage of ATP sensor FRET efficiency distribution after $10 \mu \mathrm{M}$ antimycin A addition in $a p G a l^{t s}>C i^{D N}$ wings. (C) Mean FRET efficiency measured over time in the dorsal and ventral compartments in apGal $l^{t s}>C i^{D N}$ wings. Shaded regions indicate SD; solid lines illustrate a fit of the mean data. Black arrows indicate the addition of the drug, and small colored dots represent the averages 
at each timepoint. (D, E) Fit parameters of individual time traces for dorsal and ventral compartments. Black lines connect the corresponding regions of the same disc. $* *=p$-value $<0.01, * * *=p$-value $<$ 0.001 using a Kruskal-Wallis test $(\mathrm{n}=13)$.

\section{MATERIALS AND METHODS:}

\section{Fly stocks, husbandry and genetics}

The following fly stocks were used: Wild-type Oregon-R (BDSC \#5), ap-Gal4 (BDSC \#3041), tub-Gal80 ${ }^{t s}$ (BDSC \#7017 or 1019), UAS-Ptc ${ }^{R N A i}$ (BDSC) and UAS-Ci $i^{D N}$ $\left(\mathrm{Ci}^{\text {Cell }}\right)$ (Méthot and Basler, 1999). All flies and larvae were raised on a standard food containing cornmeal, agar, malt, sugar beet syrup, brewery yeast, propionic acid and soy flour under a 12 $\mathrm{hr}$ light/dark cycle. All the knockdown and over-expression experiments for immunostainings and FRET analysis were performed with apGal4-Gal80 $0^{t s}$ (used here as apGalts). 20-30 female ap Gal ${ }^{\text {ts }}$ flies were crossed to male $U A S-P t c^{R N A i}$ or $U A S-C i^{D N}$ flies in a 3:1 ratio in a normal food vial. Flies were allowed to lay eggs in this vial in a $20^{\circ} \mathrm{C}$ incubator or water bath and then were transferred to a new vial every day. Larval growth took place at $20^{\circ} \mathrm{C}$ for one week, and then larvae were transferred to $30^{\circ} \mathrm{C}$ for $24 \mathrm{hr}$. Then, upcrawling larvae were selected and both sexes were dissected. In all cases, the necessary controls (outcrosses with wild-type Oregon-R flies) were handled in the same way.

\section{Generation of transgenic lines}

The FRET sensor ubi-AT1.03NL was generated as described in (Spannl et al., 2020). Additionally, apGal ${ }^{\text {ts }}$ was introduced into the background of the ubi-AT1.03NL flies and was used for the spatial-temporal overexpression of $P t c^{R N A i}$ and $C i^{D N}$.

\section{Imaging FRET-based ATP sensor in wing explants}

Wing discs from upcrawling third-instar larvae were dissected in full medium (Grace's medium (Sigma G9771) supplemented with 5\% FBS (ThermoFischer/Invitrogen 10270098) and $20 \mathrm{nM}$ of 20-hydroxyecdysone (Sigma H5142)(Dye et al., 2017) within $10 \mathrm{~min}$ and mounted as in (Spannl et al., 2020): basal side up on glass-bottom dishes (MatTek Corporation, \#P35G-1.0-20 C) with a double-sided tape spacer and immobilized with a Whatman ${ }^{\mathrm{TM}}$ Cyclopore $^{\mathrm{TM}}$ track-etched polycarbonate membrane filter (GE Healthcare Life Sciences, \#7062-2513). Then, $1 \mathrm{ml}$ of full medium was added and samples were transferred to the microscope. All experiments were performed at $25{ }^{\circ} \mathrm{C}$, including the ones with $P t c^{R N A i}$, as we assume that new expression of Ptc will require longer than the $2 \mathrm{hr}$ experiment.

For the experiments with metabolic drugs, $1 \mathrm{ml}$ of full medium with $2 \mathrm{X}$ concentration of antimycin A (Sigma-Aldrich \#A8674), 3-bromopyruvate (Sigma Aldrich \#16490) or 2deoxy-D-glucose (CARLROTH \#CN96.3) was further added to the dish on the microscope using a hole on the lid. Drugs were added (shown with a black arrow in figures) either immediately after acquiring the first time point (time point $0 \mathrm{~min}$ ) or after $10 \mathrm{~min}$. Final concentrations are described in the figure legends. 
Images of the wing pouch were acquired on an Olympus IX81 microscope equipped with CSU-W1 spinning disk (Yokogawa), Andor iXon Ultra 888, Monochrome EMCCD camera, Prior PRO SCAN III, Prior NanoScanZ and an incubation chamber to ensure stable temperature $\left(25^{\circ} \mathrm{C}\right)$. For all experiments a $60 \times$ silicone oil immersion objective lens was used (UPLSAPO60xS2, NA = 1.3). Wing discs were excited with a $445 \mathrm{~nm}$ laser twice in a sequential manner. Emission of mse-CFP was collected upon first excitation using an HQ 480/40 bandpass filter, and emission of cpVenus-FRET was collected using an HQ 542/27 filter. The bleedthrough of mse-CFP into the HQ 542/27 filter was estimated by exciting wing discs expressing ubi-Gal4-driven CFP-tagged human cytoplasmic $\beta$-actin (BDSC \#7064) and acquiring images through an HQ 480/40 filter $\left(\mathrm{I}_{\mathrm{D}}\right)$ and its bleedthrough in an HQ 542/27 filter $\left(\mathrm{I}_{\mathrm{bth}}\right)$. The fraction of FRET intensity contributed by bleed-through is given by:

$$
\beta=\frac{I_{b t h}}{I_{D}}
$$

\section{Analysis of FRET-based ATP sensor imaging}

FRET data were analyzed as previously described (Spannl et al., 2020). Briefly, a custom-written MATLAB (MathWorks) script was used to estimate the FRET efficiency from the fluorescence images after smoothening both donor and FRET images using a $5 \times 5$ averaging kernel (Spannl et al., 2020). Donor $\left(I_{D}\right)$ and FRET $\left(I_{F}\right)$ images were background subtracted, and the FRET intensity was corrected for bleedthrough as:

$$
I_{F R E T}=I_{F}-\beta I_{D}
$$

Finally, the FRET efficiency $(\eta)$ was calculated as:

$$
\eta=\frac{I_{F R E T}}{I_{D}+I_{F R E T}}
$$

To separately quantify ATP dynamics in regions of the wing disc (Fig 1-3), the MATLAB script was modified by the Image Analysis Clinic of MPI-CBG to calculate FRET efficiency in user-defined circular ROIs of $20 \mu \mathrm{m}$ diameter (Fig S1H). Using the maximum intensity projection of the cpVenus channel (not the FRET), it is possible to discern AP and DV boundary regions by eye (Fig S1G). The AP boundary appears as a stripe of slightly lower intensity, and the DV boundary lies in the middle of two strips of higher intensity. Using the MATLAB script, we define circular 9 ROIs (Fig S1H). The organizer region of the AP boundary was defined as the average of three circular ROIs in the AP compartment boundary, whereas the organizer region of the DV boundary was defined as the average of three circular ROIs in the DV compartment boundary. AP and DV boundaries shared the central ROI. Consequently, the average FRET efficiency of both organizer regions was estimated from these five circular ROIs described in the AP and DV compartment boundaries. Non-organizer regions were defined as the average of four circular ROIs laying outside of the AP and DV compartment boundaries (Fig S1G-H). To quantify FRET efficiency over the entire dorsal or ventral 
compartment of each disc (Fig 4, S3, S4A and S4G-K), a freehand tool was used to select these regions and calculate their average FRET efficiency.

\section{Empirical fit of ATP decline}

Data from the FRET efficiency decline over time upon addition of metabolic drugs were fit using MATLAB (R2021a, The MathWorks Inc.) with the following equation:

$$
f(t)=\text { base }+\frac{\text { max }- \text { base }}{\left(1+\frac{t_{1 / 2}}{t}\right)^{n}}
$$

where $t_{1 / 2}$ and $\mathrm{n}$ are the two fit parameters corresponding to the half-life (loss of 50\% of FRET efficiency) and the Hill coefficient, respectively. Base and max were incorporated to account for the fact that our samples have variable starting and ending values of FRET. The base was set to be 0.2827 , as this is the lowest reliable recorded FRET efficiency value derived from the z-stack of a morphologically healthy, unperturbed wing disc expressing ubiquitously the AT1.03RK sensor, as explained in Fig S1F. Max is also a fit parameter that never exceeded 0.7, which is the highest reliable mean FRET efficiency value that we have recorded, derived from morphologically healthy, unperturbed wing discs expressing ubiquitously the AT1.03NL sensor. Graphs were generated from the same script.

\section{Measurement of bulk ATP levels using a luciferase-based assay}

Bulk levels of ATP were measured using a luciferase-based biochemical assay (ATPlite Luminescence Assay System, PerkinElmer). Wing discs from third instar upcrawling larvae were dissected in culture medium, washed with PBS within seconds, suspended in $20 \mu \mathrm{l}$ of PBS, and added to wells of white polysterene flat bottom 96-well assay plates (Costar® 3917) containing $80 \mu \mathrm{l}$ of PBS (to make a final volume of $100 \mu \mathrm{l}$ in PBS). Specifically for Fig S2A$\mathrm{B}$, experiments were done with paired discs from each larva (right and left). One disc was treated with either 3BP or antimycin A (Fig S2A) or 3BP and 2DG (Fig S2B) and the other was mock-treated, serving as a control. The blank control was $100 \mu \mathrm{l}$ of PBS. Samples were lysed by the addition of $50 \mu \mathrm{l}$ of mammalian cell lysis solution followed by shaking at $700 \mathrm{rpm}$ for $10 \mathrm{~min}$. Luciferase substrate solution was added in a volume of $50 \mu \mathrm{l}$, and the samples were shaken at $700 \mathrm{rpm}$ for $5 \mathrm{~min}$. After $10 \mathrm{~min}$ of incubation in the dark, luciferase activity was measured using a Perkin Elmer Envision plate reader. To estimate concentration, a standard curve of luciferase activity was generated using a serial dilution of a $10 \mathrm{mM}$ ATP stock solution.

To calculate the average wing disc volume, dissected wing discs from upcrawling larvae expressing the AT1.03NL FRET sensor were mounted and placed on the Olympus IX81 microscope as described earlier (Imaging FRET-based ATP in wing explants). Wing discs were excited with a $445 \mathrm{~nm}$ laser and emission of cpVenus-FRET was collected using an HQ 542/27 filter. Serial acquisition of images every $0.5 \mu \mathrm{m}$ from the most apical to the most basal disc part resulted in a z-stack including the entire wing disc. The Image Analysis Clinic provided a FIJI 
macro that processes the z-stacks based on the fluorescence intensity (emission collected using the HQ 542/27 filter) and calculates the wing disc volume. The volume of 14 wing discs was used to estimate the average wing disc volume. To estimate the intracellular ATP concentration in approximation, the amount of ATP measured from single-discs luciferase assays was divided by the average wing disc volume.

\section{Immunofluorescence}

Wing discs from upcrawling third instar larvae were dissected in PBS, fixed in $4 \%$ paraformaldehyde (PFA) for $20 \mathrm{~min}$, and rinsed three times in PBS. Wing discs were then permeabilized with $0.05 \%$ Triton X-100 in PBS (PBX) twice for $10 \mathrm{~min}$, blocked for $45 \mathrm{~min}$ in $\mathrm{PBX}+1 \mathrm{mg} / \mathrm{ml} \mathrm{BSA}+250 \mathrm{mM} \mathrm{NaCl}(\mathrm{BBX} 250)$, and incubated overnight with the primary antibody in PBX $+1 \mathrm{mg} / \mathrm{ml} \mathrm{BSA}(\mathrm{BBX})$ at $4{ }^{\circ} \mathrm{C}$. After washing twice for $20 \mathrm{~min}$ in BBX, wing discs were blocked for 45 min in the blocking solution (BBX $+4 \%$ normal goat serum) and incubated for 2-3 hr with the secondary antibody in the blocking solution. Afterwards, the wing discs were rinsed two times and washed three times within $45 \mathrm{~min}$ in PBX and the same in PBS. Finally, wing discs were mounted in VectaShield ${ }^{\circledR}$ (Vector Labs, \#H-1000). Secondary antibodies conjugated with Alexa Fluor ${ }^{\circledR} 488$ and 555 were diluted 1:1000 and Alexa Fluor ${ }^{\circledR}$ 647 were diluted 1:500 (ThermoFisher Scientific). Primary antibodies used:

\begin{tabular}{|c|c|c|c|c|}
\hline Antigen & Host species & Dilution used & Origin & Genotype \\
\hline Ptc & mouse & $1: 100$ & DSHB, AB_528441 & $a p G a l^{t s}>P t c^{R N A i} / W T$ \\
\hline $\mathrm{Ci}_{155}$ & rat & $1: 30$ & DSHB, AB_2109711 & $a p G a l^{t s}>P t c^{R N A i} / W T$ \\
\hline PH3 & rabbit & $1: 500$ & Cell signaling \#9701S & $a p G a l^{t s}>P_{t c}^{R N A i} / W T$ \\
\hline
\end{tabular}

Images were acquired using a Zeiss LSM700 inverted confocal microscope equipped with a Zeiss Axio Observer.Z1, a motorized stage Maerzhauser Wetzler Gmbh EK 130×85 mot. Tango CZ EMV, a 25×/0.8 LCI Plan-Neofluor, W/Glyc/Oil objective (Zeiss) and 2 PMT. Both samples and controls were dissected, fixed, stained, and imaged in parallel so that the reagents and handling conditions were always the same. Fiji (Schindelin et al., 2012) was used for image processing, orienting, and segmenting. Z-stacks of PH3 were max-projected and segmented using the Weka segmentation plugin (Arganda-Carreras et al., 2017) as previously described (Dye et al., 2017). Statistical analyses and plots were made using GraphPad Prism 9.

To confirm the colocalization of the FRET pattern close to the AP boundary with the Ptc stripe (Fig S1I-J), FRET efficiency was calculated in wing discs while treated with $10 \mu \mathrm{M}$ antimycin A for up to $60 \mathrm{~min}$, as previously described (Imaging FRET-based ATP in wing disc explant). At the end of $60 \mathrm{~min}$, the wing discs were fixed on site with $4 \%$ PFA, and the glass bottom plates with the samples were removed so that immunostainings for the detection of Ptc with Alexa Fluor ${ }^{\circledR} 647$ could continue, as described above. At the end of the immunostainings, the samples were taken back to the spinning disc microscope. Wing discs were excited with a $638 \mathrm{~nm}$ laser, and emission of Alexa Fluor® 647 was collected with a HQ 685/40 filter. Afterwards, images from both FRET and immunofluorescence experiments were compared for the same wing discs (Fig S1I-J). 


\section{Statistical analyses}

442

Statistical analyses were performed using GraphPad Prism 9 or MATLAB (R2021a, The MathWorks Inc.). For statistical significance, Kruskal-Wallis, paired t-tests, Mann Whitney tests, one-way ANOVA with Bonferroni correction and unpaired t-tests with Welch's correction were performed as listed in the figure legends for each experiment. The use of either parametric or non-parametric statistical analysis was determined by the normal or not normal distribution of data, respectively.

\section{ACKNOWLEDGEMENTS:}

This work was supported by funding from the Max Planck Society and the Deutsche Forschungsgemeinschaft (SFB/TRR83). We thank the facilities of the MPI-CBG, specifically the Light Microscopy Facility, Scientific Computing (in particular Gayathri Nadar \& Noreen Walker from the Image Analysis Clinic), and the fly facility (Sven Ssykor and Cornelia Maas). We also thank Suhrid Ghosh, Romina Piscitello-Gómez, Jonathan Rodenfels, and Michele Solimena for critical comments on the manuscript prior to submission. We heartfully thank the members of the Eaton lab, in particular Ali Mahmoud for excellent lab management and support. Lastly, we dedicate this paper to our inspiring mentor and co-author, Professor Dr. Suzanne Eaton, who tragically passed away near the conclusion of this project.

\section{AUTHOR CONTRIBUTIONS:}

IN performed experiments and analyzed data. KVI established the FRET acquisition methods, and JMIA developed the algorithmic tools used by IN to fit and analyze the FRET data. SE conceived the study. IN, SE, NAD and AN designed the study and analyzed data. IN and NAD wrote the manuscript with discussions and feedback from all authors.

\section{CONFLICT OF INTEREST}

The authors declare no conflicts of interest.

\section{REFERENCES}

Affolter, M. and Basler, K. (2007) 'The Decapentaplegic morphogen gradient: from pattern formation to growth regulation.', Nature reviews. Genetics, 8(9), pp. 663-674. doi: $10.1038 / \mathrm{nrg} 2166$.

Arganda-Carreras, I. et al. (2017) 'Trainable Weka Segmentation: a machine learning tool for microscopy pixel classification', Bioinformatics, 9, pp. 676-682. doi: 10.1093/bioinformatics/btx180.

Basler, K. and Struhl, G. (1994) 'Compartment boundaries and the control of Drosopfiffa limb pattern by hedgehog protein', Nature. Nature Publishing Group, 368(6468), pp. 208214. doi: $10.1038 / 368208 \mathrm{a} 0$. 
484 Bawa, S. et al. (2020) 'Drosophila TRIM32 cooperates with glycolytic enzymes to promote 485 cell growth', eLife. eLife Sciences Publications Ltd, 9. doi: 10.7554/eLife.52358.

486 Beira, J. V. and Paro, R. (2016) 'The legacy of Drosophila imaginal discs', Chromosoma, pp. 487 573-592. doi: 10.1007/s00412-016-0595-4.

488 Brand, A. H. and Perrimon, N. (1993) 'Targeted gene expression as a means of altering cell 489 fates and generating dominant phenotypes', Development. Development, 118(2), pp. 401490 415. doi: 10.1242/dev.118.2.401.

491 Bulusu, V. et al. (2017) 'Spatiotemporal Analysis of a Glycolytic Activity Gradient Linked to 492 Mouse Embryo Mesoderm Development', Developmental Cell. Cell Press, 40(4), pp. 331493 341.e4. doi: 10.1016/j.devcel.2017.01.015.

Denef, N. et al. (2000) 'Hedgehog induces opposite changes in turnover and subcellular localization of patched and smoothened', Cell. Elsevier, 102(4), pp. 521-531. doi: 10.1016/S0092-8674(00)00056-8.

Dye, N. A. et al. (2017) 'Cell dynamics underlying oriented growth of the drosophila wing imaginal disc', Development (Cambridge). Company of Biologists Ltd, 144(23), pp. 44064421. doi: 10.1242/dev.155069.

Gershon, T. R. et al. (2013) 'Hexokinase-2-mediated aerobic glycolysis is integral to cerebellar neurogenesis and pathogenesis of medulloblastoma', Cancer \& Metabolism. Springer Nature, 1(1). doi: 10.1186/2049-3002-1-2.

Greenwald, E. C., Mehta, S. and Zhang, J. (2018) 'Genetically encoded fluorescent biosensors illuminate the spatiotemporal regulation of signaling networks', Chemical Reviews. American Chemical Society, 118(24), pp. 11707-11794. doi: 10.1021/acs.chemrev.8b00333.

Hariharan, I. K. (2015) 'Organ Size Control: Lessons from Drosophila', Developmental Cell, 34(3), pp. 255-265. doi: 10.1016/j.devcel.2015.07.012.

508 Ingham, P. W. and McMahon, A. P. (2001) 'Hedgehog signaling in animal development: 509 Paradigms and principles', Genes and Development. Cold Spring Harbor Laboratory Press, 510 pp. 3059-3087. doi: 10.1101/gad.938601.

511 De La Cova, C. et al. (2014) 'Supercompetitor status of drosophila Myc cells requires p53 as 512 a Fitness sensor to reprogram metabolism and promote viability', Cell Metabolism. Elsevier, 513 19(3), pp. 470-483. doi: 10.1016/j.cmet.2014.01.012.

514 Lunt, S. Y. and Vander Heiden, M. G. (2011) 'Aerobic glycolysis: Meeting the metabolic 515 requirements of cell proliferation', Annual Review of Cell and Developmental Biology, 27, pp. 516 441-464. doi: 10.1146/annurev-cellbio-092910-154237.

517 Di Magno, L. et al. (2014) 'Druggable glycolytic requirement for Hedgehog-dependent 518 neuronal and medulloblastoma growth', Cell Cycle. Landes Bioscience, 13(21), pp. 3404519 3413. doi: 10.4161/15384101.2014.952973.

520 Méthot, N. and Basler, K. (1999) 'Hedgehog controls limb development by regulating the activities of distinct transcriptional activator and repressor forms of cubitus interruptus', Cell. Elsevier B.V., 96(6), pp. 819-831. doi: 10.1016/S0092-8674(00)80592-9. 
Petrova, R. and Joyner, A. L. (2014) 'Roles for Hedgehog signaling in adult organ homeostasis and repair', Development (Cambridge). Company of Biologists Ltd, pp. 34453457. doi: 10.1242/dev.083691.

Restrepo, S., Zartman, J. J. and Basler, K. (2014) 'Coordination of patterning and growth by the morphogen DPP', Current Biology. Cell Press, pp. R245-R255. doi: 10.1016/j.cub.2014.01.055.

Rogers, K. W. and Schier, A. F. (2011) 'Morphogen gradients: From generation to interpretation', Annual Review of Cell and Developmental Biology. Annual Reviews, 27, pp. 377-407. doi: 10.1146/annurev-cellbio-092910-154148.

Schindelin, J. et al. (2012) 'Fiji: an open-source platform for biological-image analysis.', Nature methods. NIH Public Access, 9(7), pp. 676-82. doi: 10.1038/nmeth.2019.

Slaninova, V. et al. (2016) 'Notch stimulates growth by direct regulation of genes involved in the control of glycolysis and the tricarboxylic acid cycle.', Open biology. Royal Society Journals, 6(2), p. 150155. doi: 10.1098/rsob.150155.

Spannl, S. et al. (2020) 'Glycolysis regulates Hedgehog signalling via the plasma membrane potential', The EMBO Journal. EMBO, 39(21), p. e101767. doi: 10.15252/embj.2019101767.

Tabata, T. and Kornberg, T. B. (1994) 'Hedgehog is a signaling protein with a key role in patterning Drosophila imaginal discs', Cell. Cell Press, 76(1), pp. 89-102. doi: 10.1016/00928674(94)90175-9.

542 Teperino, R. et al. (2012) 'Hedgehog partial agonism drives warburg-like metabolism in muscle and brown fat', Cell. Elsevier B.V., 151(2), pp. 414-426. doi: 10.1016/j.cell.2012.09.021.

545 Tsuyama, T. et al. (2013) 'In vivo fluorescent adenosine 5'-triphosphate (ATP) imaging of

546 Drosophila melanogaster and Caenorhabditis elegans by using a genetically encoded

547 fluorescent ATP Biosensor optimized for low temperatures', Analytical Chemistry. American

548 Chemical Society, 85(16), pp. 7889-7896. doi: 10.1021/ac4015325.

549 Del Valle Rodríguez, A., Didiano, D. and Desplan, C. (2012) 'Power tools for gene 550 expression and clonal analysis in Drosophila’, Nature Methods. NIH Public Access, pp. 47551 55. doi: 10.1038/nmeth. 1800.

552 Volkenhoff, A. et al. (2018) 'Live imaging using a FRET glucose sensor reveals glucose 553 delivery to all cell types in the Drosophila brain', Journal of Insect Physiology. Elsevier Ltd, 554 106(Pt 1), pp. 55-64. doi: 10.1016/j.jinsphys.2017.07.010.

555 Wang, C.-W. et al. (2016) 'In vivo genetic dissection of tumor growth and the Warburg 556 effect', eLife, 5. doi: 10.7554/eLife.18126. 
bioRxiv preprint doi: https://doi.org/10.1101/2021.09.11.459911· this version posted September 12, 2021. The copyright holder for this preprint (which was not certified by peer review) is the author/funder, who has granted bioRxiv a license to display the preprint in perpetuity. It is made available under aCC-BY 4.0 International license.

A

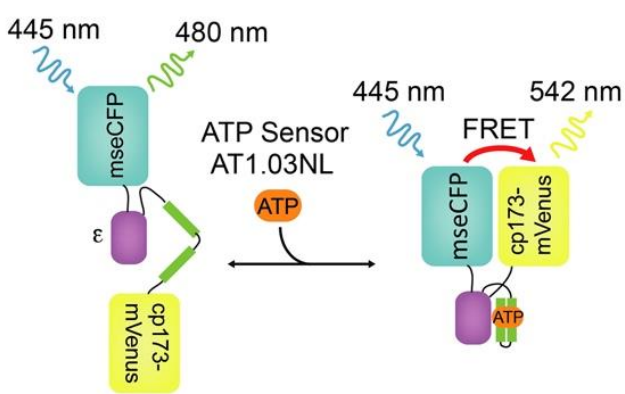

B

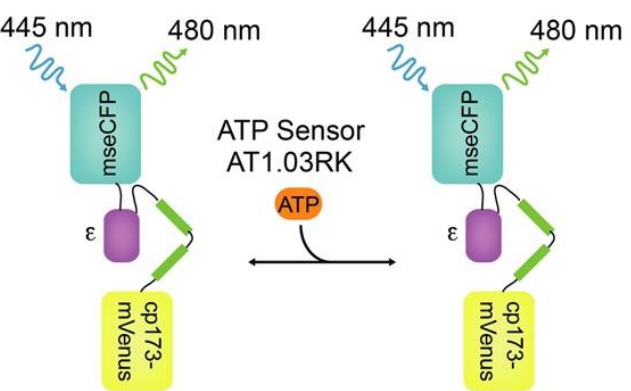

C
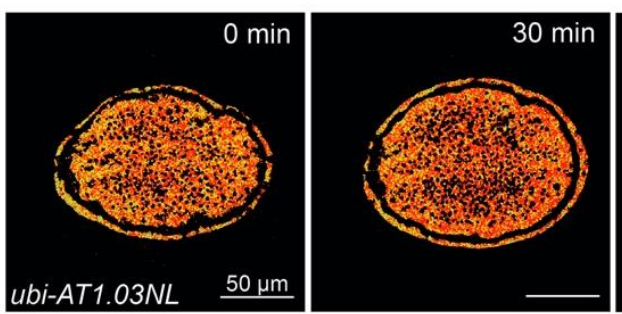

E

FRET efficiency of mock-treated wing discs
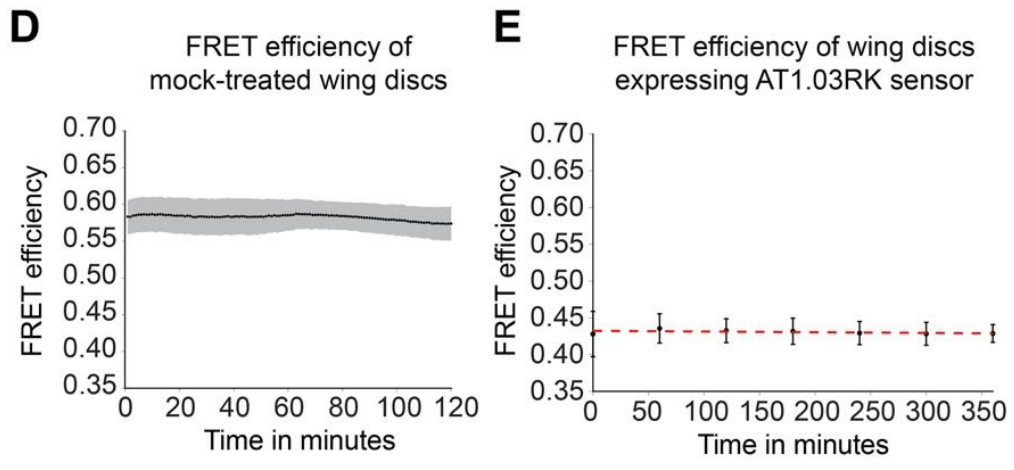
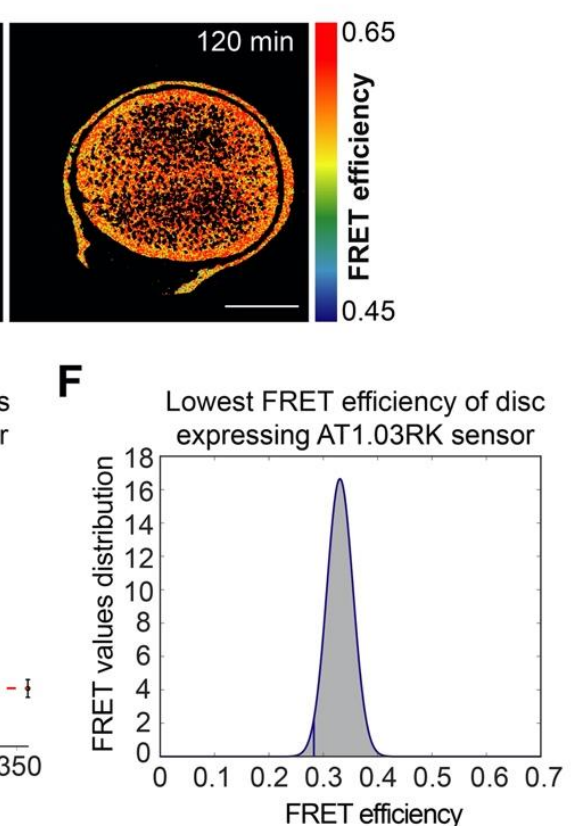

\section{G}

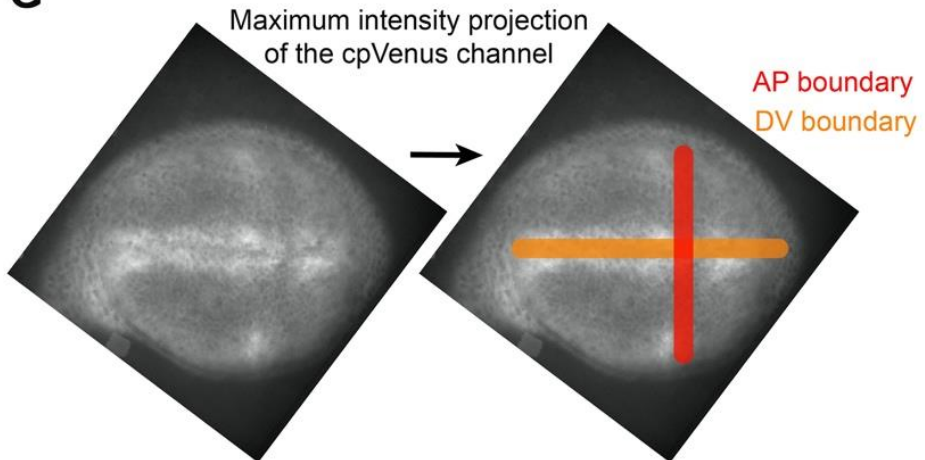

I
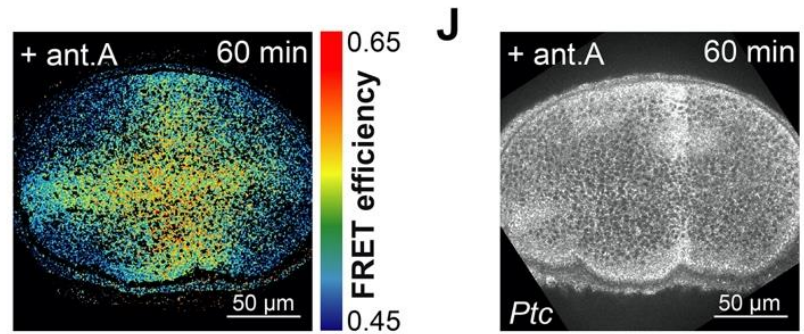

H

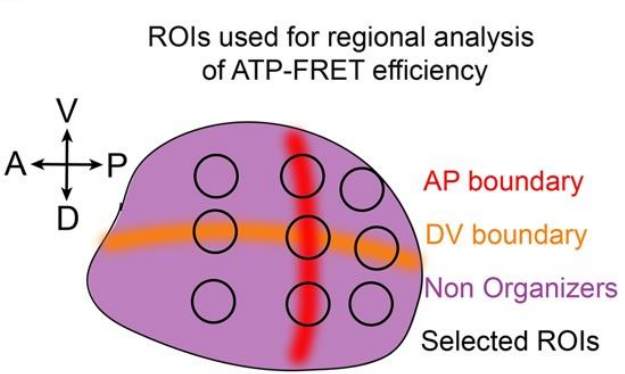

Extended data S1. ATP levels in the wing pouch are spatially uniform and stable in culture without

OxPhos inhibition. (A, B) Schematic representation of ATP-FRET sensor design (AT1.03NL) and its ATP-insensitive version (AT1.03RK). (C) Timelapse montage of ATP-FRET sensor efficiency in the wing pouch during culture for $2 \mathrm{hr}$. (D) Mean FRET efficiency in the entire pouch measured over time; gray shade indicates $\mathrm{SD}$, and small black dots represent the averages at each time point $(\mathrm{n}=9)$. (E) Mean FRET efficiency in the entire pouch expressing AT1.03RK (red dashed line). The black error bars 
565 represent SD for each time point $(n=7)$. (F) Histogram showing the distribution of FRET efficiency 566 values across a 70-plane z-stack of a morphologically healthy, unperturbed wing disc ubiquitously expressing the AT1.03RK sensor: gray area corresponds to the FRET values, and the blue curved line shows a Gaussian fit. The lowest FRET efficiency value used for data fitting was 0.2827 (blue vertical line), defined as the mean FRET efficiency minus twice the standard deviation. (G) AP and DV boundary regions are discernible with a maximum intensity projection of the cpVenus channel. The AP boundary appears as a stripe of slightly lower intensity, and the DV boundary lies in the middle of two strips of higher intensity. (H) Schematic indicating the location of the ROIs that were used to calculate mean FRET efficiency in different wing pouch regions. The three ROIs in the AP boundary or in the DV boundary were averaged together to calculate the mean FRET efficiency of the AP boundary and DV boundary, respectively. The organizer region was measured as the average of all five of these ROIs (AP+DV boundaries). The non-organizer region corresponds to the remaining four ROIs outside of the AP+DV boundaries. (I) Spatial pattern of FRET efficiency after 60 min of $10 \mu \mathrm{M}$ antimycin A (ant. A) exposure, and (J) Ptc expression in the same disc after fixation and immunofluorescence. 
A

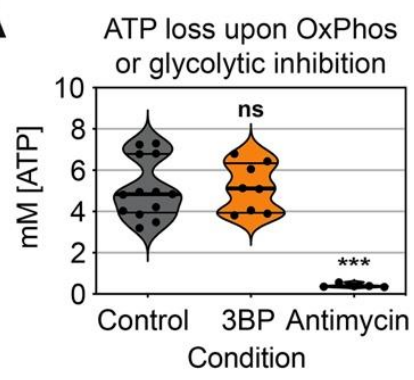

B

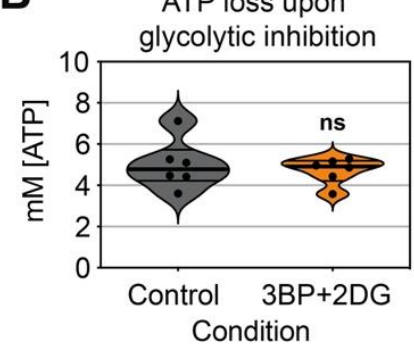

C Time course of ATP loss

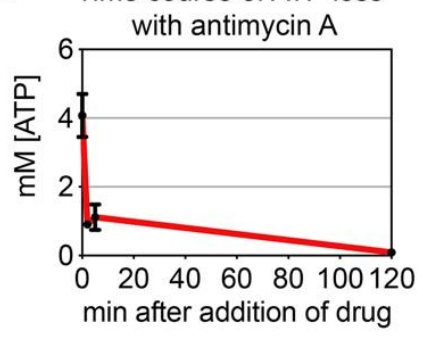

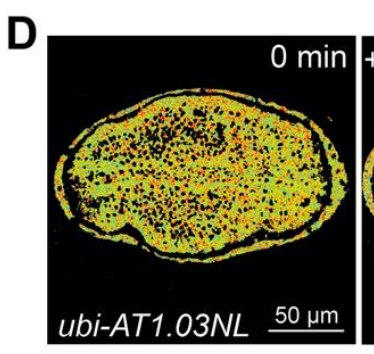

E

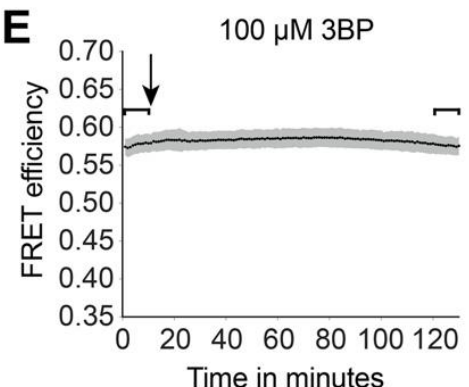

Time in minutes

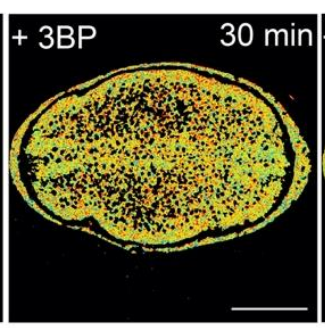

$\mathbf{F}$

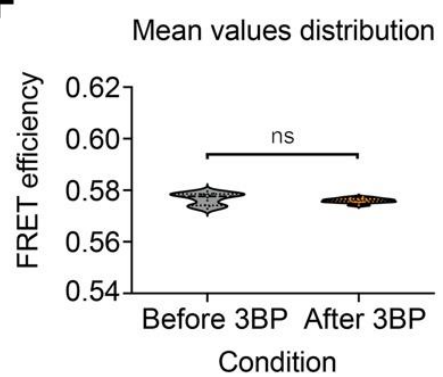

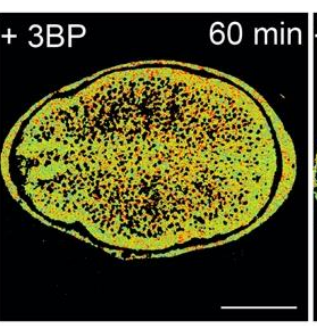

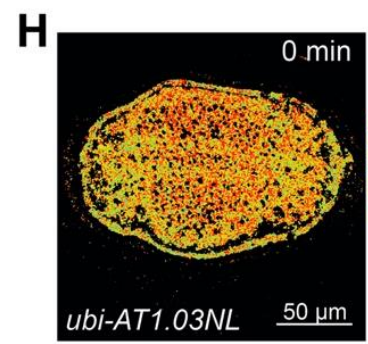

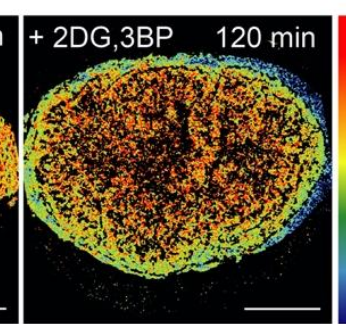

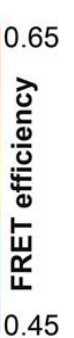

Extended data S2. Glycolysis inhibitors alone do not significantly affect ATP levels. (A) ATP levels measured using a luminescence-based biochemical assay from single discs after a $2 \mathrm{hr}$ treatment with either $50 \mu \mathrm{M} 3 \mathrm{BP}(\mathrm{n}=8)$ or $10 \mu \mathrm{M}$ antimycin $\mathrm{A}(\mathrm{n}=5)$ compared to untreated discs $(\mathrm{n}=13)$. *** $=$ p-value $<0.001, n s=$ not significant using either Mann-Whitney test (control vs 3BP) or unpaired t-test (control vs antimycin A). (B) ATP levels of single discs after $1 \mathrm{hr}$ of treatment with $50 \mu \mathrm{M} 3 \mathrm{BP}+50$ $\mathrm{mM} 2 \mathrm{DG}(\mathrm{n}=6)$ compared to untreated discs $(\mathrm{n}=6) . \mathrm{ns}=$ not significant using a paired $\mathrm{t}$-test. (C) Decline of ATP levels upon addition of $100 \mu \mathrm{M}$ antimycin A for varying lengths of time ( $\mathrm{n}=10$ for $0 \mathrm{~min}, \mathrm{n}=3$ for $2 \mathrm{~min}$ and $5 \mathrm{~min}$ and $\mathrm{n}=4$ for $120 \mathrm{~min}$ ). (D) Timelapse montage of ATP-FRET sensor efficiency after 3BP addition. (E) Mean FRET efficiency measured over time in the entire wing pouch upon addition of 3BP. Brackets include the mean FRET values before and $2 \mathrm{hr}$ after 3BP addition (1-10 min and 121-130 min respectively) whose distribution was compared using (F) an unpaired t-test (ns = not significant $\mathrm{p}$-value, $\mathrm{n}=11$ ) including $(\mathrm{G})$ Welch's correction and estimation plot ( $\mathrm{n}=11$ for each group). (H) Timelapse montage of ATP-FRET sensor efficiency after 3BP+2DG addition. (I) Mean FRET efficiency measured over time in the entire wing pouch upon addition of 2DG+3BP; (E, I) gray shade indicates SD ( $n=11$ in (E) and $n=9$ in (I)). Black arrows indicate the addition of the drugs, and small black dots represent the averages at each timepoint. 
A

Wing disc pouch

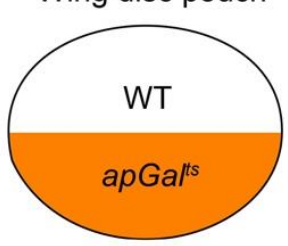

C

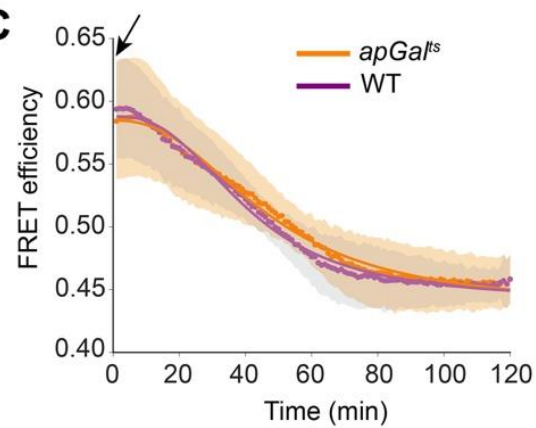

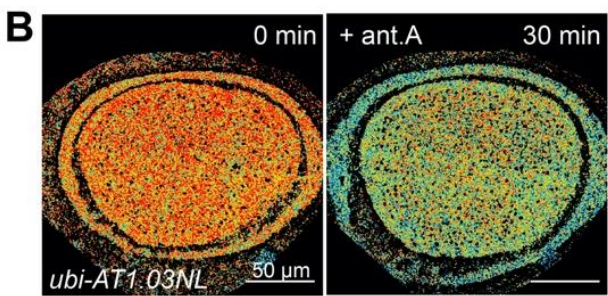

D

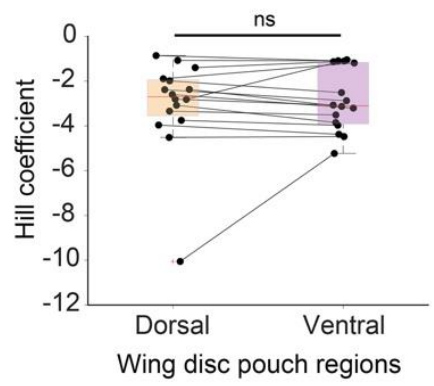

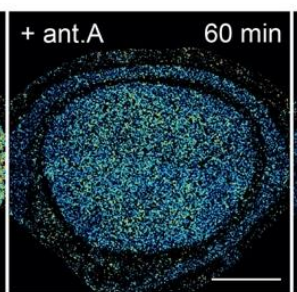

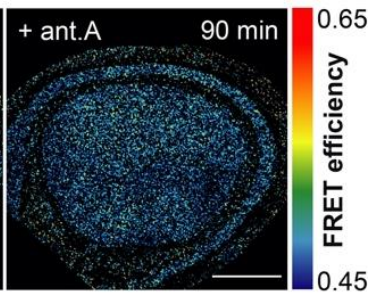

$\mathbf{E}$

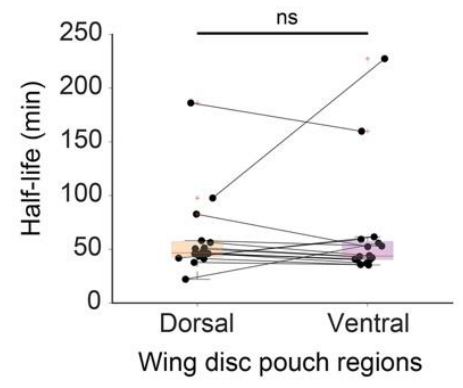

Extended data S3. OxPhos inhibition affects ATP levels similarly in the dorsal and ventral compartments of the apGal ${ }^{\text {ts }}$ genetic background (without a UAS construct). (A) Schematic representation of apGalts expression in the dorsal compartment; ventral compartment serves as an internal control (WT). (B) Mean FRET efficiency measured over time in the dorsal and ventral compartments. Shaded regions indicate SD; solid lines illustrate a fit of the mean data. (C) Timelapse montage of ATP sensor FRET efficiency in the wing pouch after $10 \mu \mathrm{M}$ antimycin A (ant.A) addition in $a p G a l^{t s}>$ WT wing discs. (D, E) Fit parameters of individual time traces for dorsal and ventral compartments. Black lines connect the corresponding regions of the same disc. ns = not significant, using Kruskal-Wallis test $(\mathrm{n}=16)$. Black arrow indicates the addition of the drugs, and small colored dots represent the averages at each timepoint. 
A

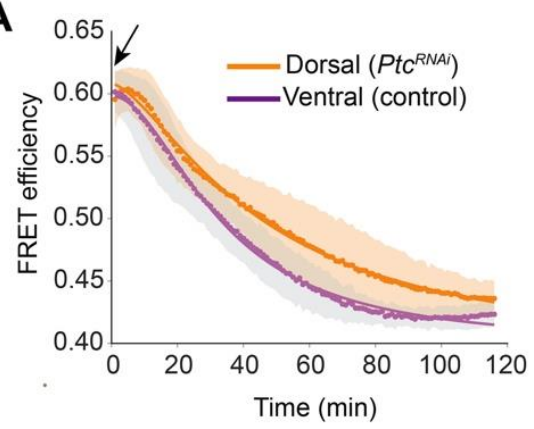

D

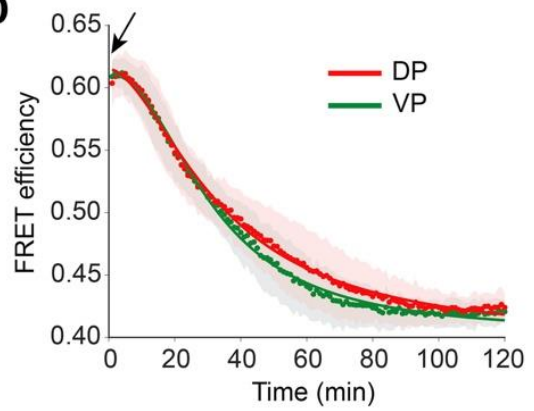

B

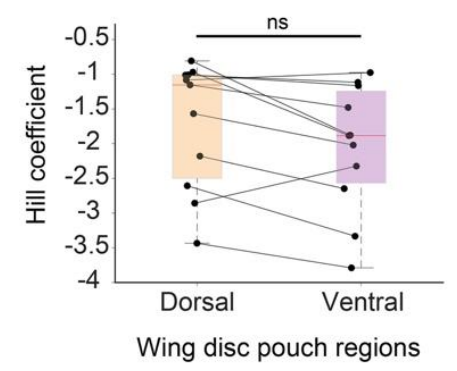

E

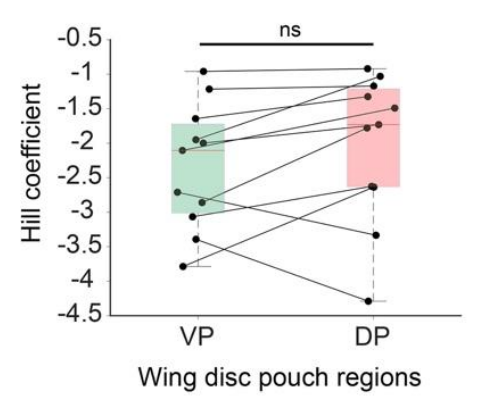

C

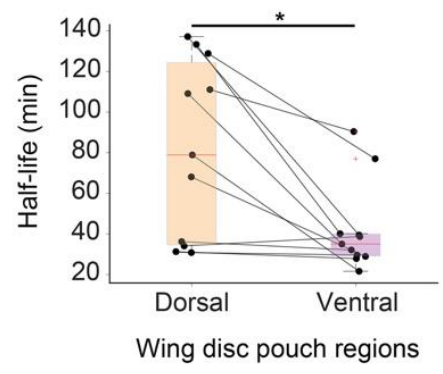

$\mathbf{F}$

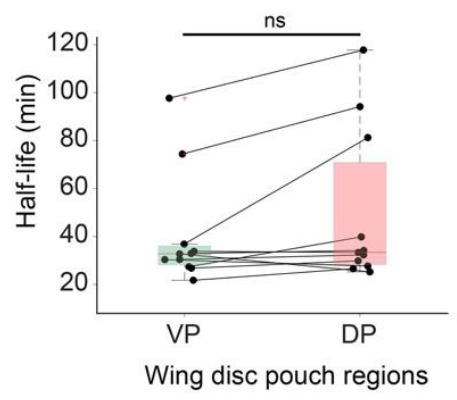

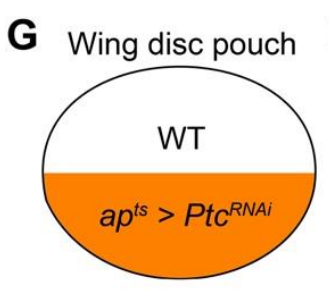
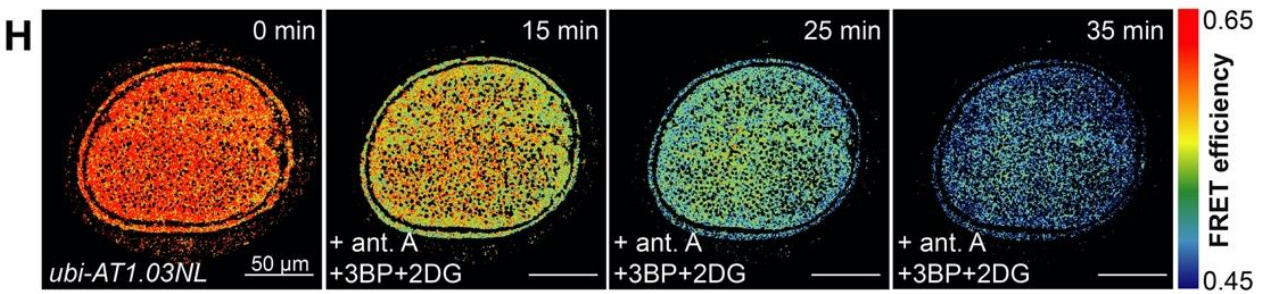

I

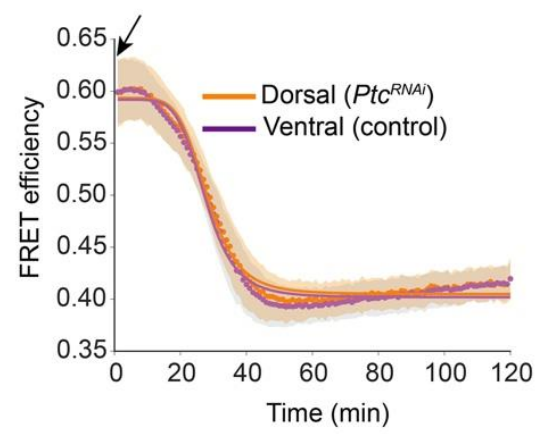

J

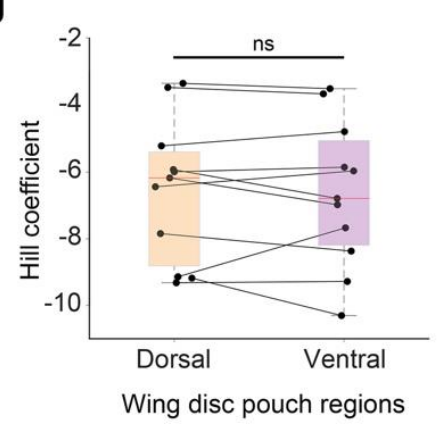

K

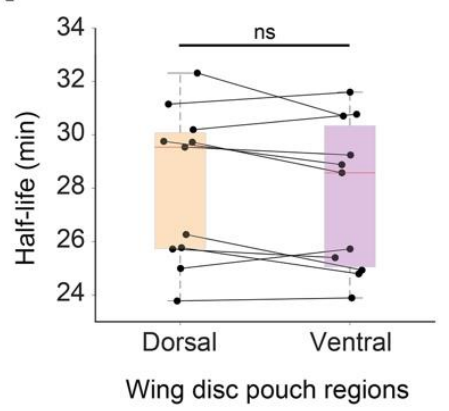

Extended data S4. Extended regional analysis of Ptc ${ }^{R N A i}$ upon OxPhos inhibition alone or combined with glycolysis inhibition. (A, D) Mean FRET efficiency measured over time in the dorsal and ventral compartments (A) or the ventral posterior (VP) and dorsal posterior (DP) sub-compartments (D) of the wing pouch in $a p G a l^{t s}>P t c^{R N A i}$ upon OxPhos inhibition. Shaded regions indicate SD; solid lines illustrate a fit of the mean data. Black arrows indicate the addition of antimycin A, and small colored dots represent the averages at each timepoint. (B-C, E-F) Fit parameters of individual time traces for the dorsal and ventral compartments (B-C) or the posterior sub-compartments (E-F). Black lines connect the corresponding regions of the same disc. $*=$ p-value $<0.05$, ns $=$ not significant, using a Kruskal-Wallis test $(\mathrm{n}=11)$. (G) Schematic representation of $a p G a l^{t s}>P t c^{R N A i}$ expression in the dorsal compartment. (H) Timelapse montage of ATP-FRET sensor efficiency after addition of antimycin A (ant. A), 3BP and 2DG. (I) Mean FRET efficiency measured over time in the dorsal and ventral compartments. Shaded regions indicate SD; solid lines illustrate a fit of the mean data. Black arrow indicates the addition of the drugs, and small colored dots represent the averages at each timepoint. (J, K) Fit parameters of individual time traces for dorsal and ventral compartments. Black lines connect the corresponding regions of the same disc. $n s=$ not significant, using Kruskal-Wallis test $(n=12)$. 

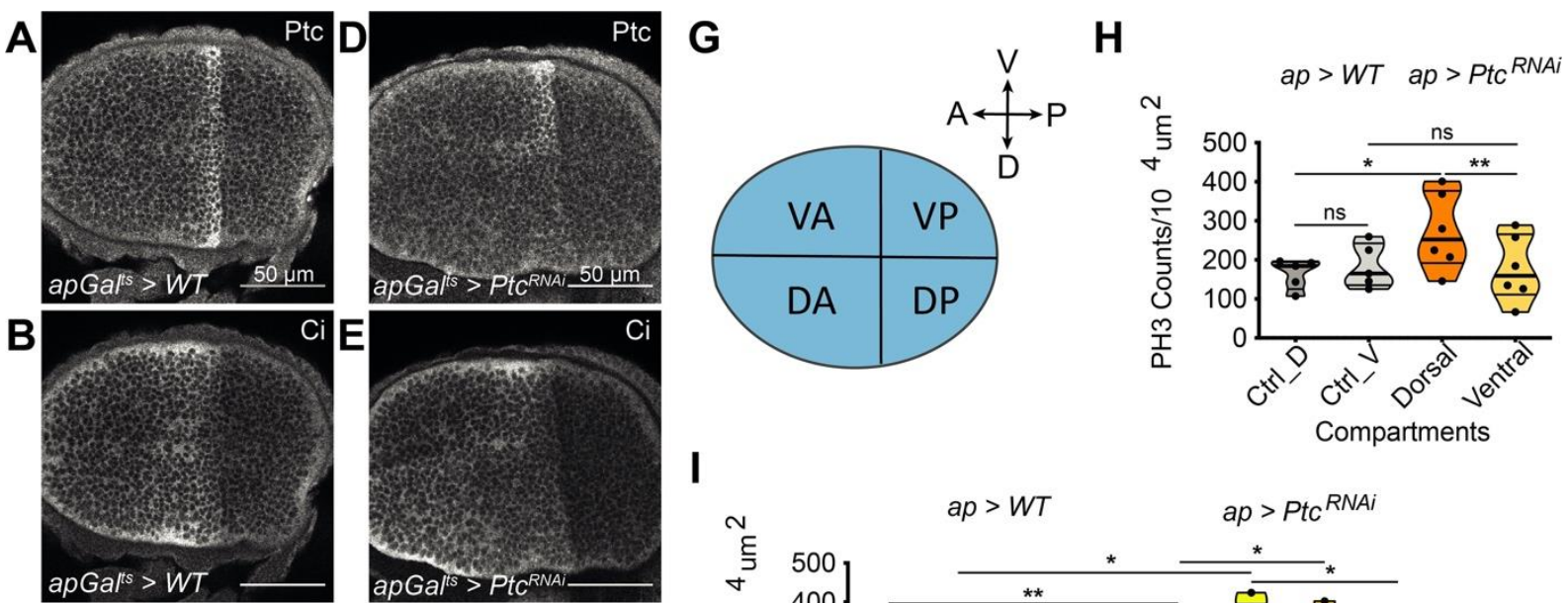

I
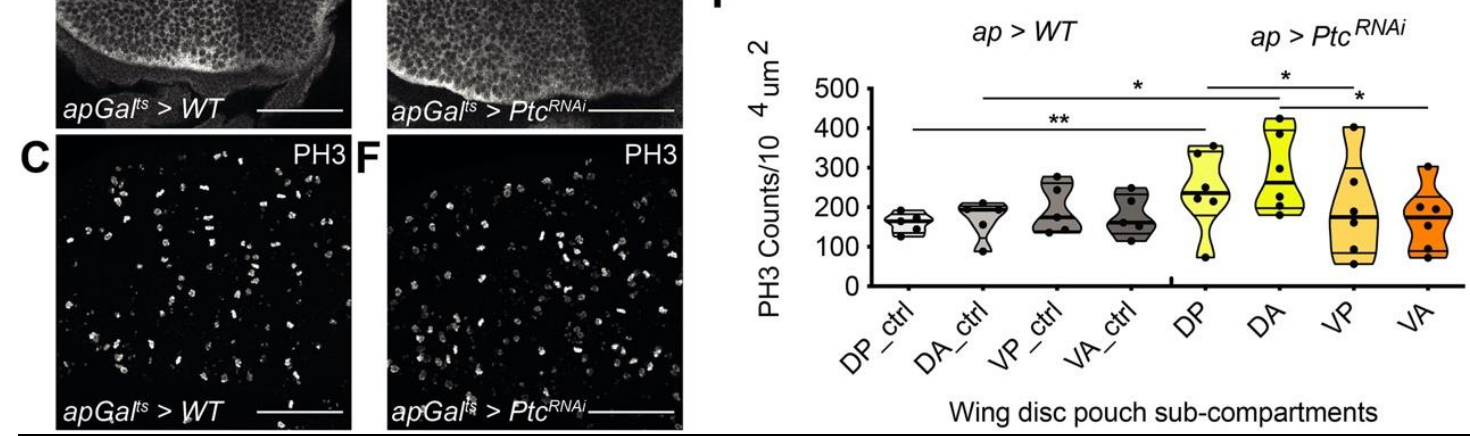

Figure S5. Upregulation of $\mathrm{Hh}$ pathway activity with $P t c^{R N A i}$ increases proliferation in both anterior and posterior compartments. Control ( $a p G a l^{t s}>W T$, A-C) or $a p G a l^{t s}>P t c^{R N A i}$ (D-F) wing pouch stained for Ptc (A, D), Ci (B, E) or phospho-histone H3 (PH3, C, F). (G) Schematic representation of wing disc sub-compartments. $\mathrm{V}=$ Ventral, $\mathrm{D}=$ Dorsal, $\mathrm{A}=$ Anterior, $\mathrm{P}=$ Posterior. $(\mathrm{H})$ Quantification of PH3-positive nuclei in the dorsal (D) and ventral (V) compartments of the pouch of control (Ctrl, $a p>W T, n=5)$ and $a p G a l^{t s}>P t c^{R N A i}(\mathrm{n}=6)$ wing discs. $*=\mathrm{p}$-value $<0.05, * *=\mathrm{p}$-value $<0.01$ using, $\mathrm{ns}$ $=$ not significant using either paired t-tests between disc compartments belonging to the same group ( $a p G a l^{t s}>$ Ptc $c^{R N A i}$ or control) or Mann-Whitney tests for different groups (Ctrl_D vs Dorsal, Ctrl_V vs Ventral). (I) Quantification of mitotic density in different compartments of the pouch in control (Ctrl, $a p>W T)$ and $a p G a l^{t s}>P t c^{R N A i}$ wing discs. $*=$ p-value $<0.05$, ** $=$ p-value $<0.01 \mathrm{using}$, ns $=$ not significant using one-way ANOVA tests with Bonferroni post-hoc correction. 